\title{
Coexistence of Competing Predators in a Chemostat
}

\author{
G. J. Butler ${ }^{1}$, S. B. Hsu ${ }^{2}$, and P. Waltman ${ }^{3}$ \\ ${ }^{1}$ Department of Mathematics, University of Alberta, Edmonton, Alberta, Canada \\ 2 Department of Applied Mathematics, National Chiao Tung University, Hsinchu, Taiwan, R.o.C. \\ ${ }^{3}$ Department of Mathematics, University of Iowa, Iowa City, IA 52242, USA
}

\begin{abstract}
An analysis is given of a mathematical model of two predators feeding on a single prey growing in the chemostat. In the case that one of the predators goes extinct, a global stability result is obtained. Under appropriate circumstances, a bifurcation theorem can be used to show that coexistence of the predators occurs in the form of a limit cycle.
\end{abstract}

Key words: Chemostat - Competition - Coexistence

\section{Introduction}

The question as to whether one prey can support two predators is an intriguing one in ecology. Although there is considerable mathematical [1], [2], [17], [19], [23] and computational [12], [17] evidence to support this possibility, the systems analyzed seem not to be directly or conveniently testable in a laboratory environment. This paper presents a mathematical analysis of the system where two predators are supported by a single prey and where the mathematical result can be tested in a common laboratory apparatus, the chemostat.

The chemostat, as used to produce a continuous supply of microorganisms, consists of a nutrient, imput at a constant rate and at a fixed concentration, flowing into a culture vessel of fixed volume; the overflow, necessary to maintain a fixed volume contains both nutrient and microorganisms. The basic equations, which go back as far as [20], are

$$
\begin{gathered}
S^{\prime}=\left(S^{(0)}-S\right) D-\frac{m x S}{\gamma(a+S)}, \\
x^{\prime}=x\left(\frac{m S}{a+S}-D\right), \quad,=\frac{d}{d t}, \\
S(0) \geqslant 0, \quad x(0) \geqslant 0 .
\end{gathered}
$$

\footnotetext{
1 Research supported by NSERC Grant A-8130

2 Research supported by National Science Council of ROC

3 Research supported by NSF Grant MCS-8120380. A portion of this research was performed while the author was a Visiting Professor at the University of Southern California, Los Angeles
} 
$S^{(0)}$ is the imput nutrient concentration rate, $D$ the dilution (or washout) rate, $m$ the maximal growth rate, $a$ the Michaelis-Menten or "half saturation" constant, and $\gamma$ a yield constant; all of these are positive quantities. $S(t)$ represents the concentration of the nutrient (or substrate), and $x(t)$, the concentration of the microorganism being cultured. A fuller discussion of the chemostat parameters and its comparison with batch culture can be found in [27].

If the culture vessel is charged with two or more types of microorganisms it is known mathematically [10], [11], [13], [24] and experimentally [9] that only one survives - the competitive exclusion principle holds.

Consider now a chemostat, as described above, and two higher level predators, $y$ and $z$, which feed exclusively on the microorganism $x$, and which compete through their consumption of this common resource. The equations take the form

$$
\begin{aligned}
& S^{\prime}=\left(S^{(0)}-S\right) D-\frac{m_{1} S x}{\gamma_{1}\left(a_{1}+S\right)}, \\
& x^{\prime}=x\left(\frac{m_{1} S}{a_{1}+S}-D-\frac{m_{2} y}{\gamma_{2}\left(a_{2}+x\right)}-\frac{m_{3} z}{\gamma_{3}\left(a_{3}+x\right)}\right), \\
& y^{\prime}=y\left(\frac{m_{2} x}{a_{2}+x}-D\right), \\
& z^{\prime}=z\left(\frac{m_{3} x}{a_{3}+x}-D\right), \\
& S(0)=S_{0} \geqslant 0, \quad x(0)=x_{0} \geqslant 0, \quad y(0)=y_{0} \geqslant 0, \quad z(0)=z_{0} \geqslant 0, \quad '=\frac{d}{d t} .
\end{aligned}
$$

The principal question is whether competitive exclusion still holds. We answer this in the negative (modulo a technical assumption, see Sect. 4). This result is important because the system (1.2) is testable in the laboratory $-S^{(0)}$ and $D$ are under the control of the experimenter and $m_{i}, a_{i}, \gamma_{i}, i=1,2,3$, are readily measurable quantities [9]. This is one reason why the chemostat plays such an important role in microbial ecology (see, for example, the four survey articles, [8], [14], [26], [27]). Based on the experiments in [6], one could suggest that $S$ is a sugar, $x$ is a bacterium, $y$ and $z$ are protozoa. Such ecosystems are also believed to play a role in waste water treatment [21].

In Sect. 2 the model is simplified by a change to nondimensional variables and a reduction of order. Section 3 analyzes a reduced system, the omega limit set in the case of competitive exclusion, and a system of interest on its own as a food chain. The local stability has been determined previously [3], [4], [22], [25], but here a global result is obtained which answers a question of Sell [22]. Section 4 deals briefly with the conditions for competitive exclusion. In Sect. 5, a bifurcation theorem is proved which shows the coexistence of the competing predators, or that competitive exclusion does not hold. This result, Theorem 5.1, follows the development in the previous work [2] with a logistically growing prey. Other results for coexistence on this latter system may be found in [1], [16], [23]. Finally in Sect. 6 , some general comments are made about generic considerations. 


\section{Simplification of the Problem}

First of all, the variables in the system (1.2) will be changed to nondimensional ones. Let

$$
\begin{aligned}
& \bar{S}=\frac{S}{S^{(0)}}, \quad \bar{x}=\frac{x}{\gamma_{1} S^{(0)}}, \quad \bar{y}=\frac{y}{\gamma_{1} \gamma_{2} S^{(0)}}, \\
& \bar{z}=\frac{z}{\gamma_{1} \gamma_{3} S^{(0)}}, \quad \bar{a}_{1}=\frac{a_{1}}{S^{(0)}}, \quad \bar{a}_{2}=\frac{a_{2}}{\gamma_{1} S^{(0)}}, \\
& \bar{a}_{3}=\frac{a_{3}}{\gamma_{1} S^{(0)}}, \quad \bar{m}_{i}=\frac{m_{i}}{D}, \quad i=1,2,3, \\
& \bar{t}=D t .
\end{aligned}
$$

Then (1.2) becomes

$$
\begin{aligned}
\bar{S}^{\prime} & =1-\bar{S}-\frac{\bar{m}_{1} \bar{x} \bar{S}}{\bar{a}_{1}+\bar{S}}, \\
\bar{x}^{\prime} & =\bar{x}\left(\frac{\bar{m}_{1} \bar{S}}{\bar{a}_{1}+\bar{S}}-1-\frac{\bar{m}_{2} \bar{y}}{\bar{a}_{2}+\bar{x}}-\frac{\bar{m}_{3} \bar{z}}{\bar{a}_{3}+\bar{x}}\right), \\
\bar{y}^{\prime} & =\bar{y}\left(\frac{\bar{m}_{2} \bar{x}}{\bar{a}_{2}+\bar{x}}-1\right), \\
\bar{z}^{\prime} & =\bar{z}\left(\frac{\bar{m}_{3} \bar{x}}{\bar{a}_{3}+\bar{x}}-1\right), \\
\prime & =\frac{d}{d \bar{t}} .
\end{aligned}
$$

Dropping the bars yields

$$
\begin{aligned}
S^{\prime} & =1-S-\frac{m_{1} x S}{a_{1}+S}, \\
x^{\prime} & =x\left(\frac{m_{1} S}{a_{1}+S}-1-\frac{m_{2} y}{a_{2}+x}-\frac{m_{3} z}{a_{3}+x}\right), \\
y^{\prime} & =y\left(\frac{m_{2} x}{a_{2}+x}-1\right), \\
z^{\prime} & =z\left(\frac{m_{3} x}{a_{3}+x}-1\right), \\
S(0) & =S_{0} \geqslant 0, \quad x(0)=x_{0} \geqslant 0, \quad z(0)=z_{0} \geqslant 0, \quad,=\frac{d}{d t}, \\
y(0) & =y_{0} \geqslant 0, \quad
\end{aligned}
$$


which is the system we wish to study. Note that the $\gamma_{i}$ 's are nondimensional, so all of the variables in (2.1) are nondimensional.

The next lemma, a "conservation law" changes the system from a four dimensional one to a three dimensional one: since every trajectory is asymptotic to its omega limit set, it is sufficient to analyze this system. Note that the positive cone in $(S, x, y, z)$-space is positively invariant under the solution map so the lemma yields the boundedness of solutions of the initial value problem (2.1).

Lemma 2.1. The omega limit set of any solution of the initial value problem (2.1) lies in the hyperplane $S+x+y+z=1$.

Proof. Let $\Sigma(t)=S(t)+x(t)+y(t)+z(t)$. Then $\Sigma^{\prime}(t)=1-\Sigma(t), \Sigma(0) \geqslant 0$ and the lemma follows.

One can use Lemma 2.1 to eliminate one variable from the system (2.1), and use will be made of this in the next two sections. In view of the lemma, trajectories which form the positive omega limit set of any solution of (2.1) are solutions of

$$
\begin{aligned}
& x^{\prime}=x\left(\frac{m_{1}(1-x-y-z)}{1+a_{1}-x-y-z}-1-\frac{m_{2} y}{a_{2}+x}-\frac{m_{3} z}{a_{3}+x}\right), \\
& y^{\prime}=y\left(\frac{m_{2} x}{a_{2}+x}-1\right), \\
& z^{\prime}=z\left(\frac{m_{3} x}{a_{3}+x}-1\right), \quad,=\frac{d}{d t}, \\
& x(0)=x_{0} \geqslant 0, \quad y(0)=y_{0} \geqslant 0, \quad z(0)=z_{0} \geqslant 0,
\end{aligned}
$$

which satisfy $0 \leqslant x, y, z \leqslant 1$.

The next simplification is to eliminate "inadequate" predators or an "inadequate" prey - those which become extinct on the level of nutrient available, or at the given dilution rate, independent of any population interactions. For example, if $m_{1} \leqslant 1$ and $x_{1}(0)>0$ then $x^{\prime}<\left(m_{1} /\left(1+a_{1}\right)-1\right) x$ and $\lim _{t \rightarrow \infty} x(t)=0$ (and consequently $\lim _{t \rightarrow \infty} y(t)=0$ and $\left.\lim _{t \rightarrow \infty} z(t)=0\right)$. Similarly if $m_{2} \leqslant 1$ or $m_{3} \leqslant 1$, the corresponding predator becomes extinct. Let $\lambda_{i}=a_{i} /\left(m_{i}-1\right), m_{i}>1$. A similar extinction result to the above follows if $\lambda_{i} \geqslant 1$. For example, suppose $\lambda_{2} \geqslant 1$. Then, since $x \leqslant 1$, we have

$$
y^{\prime}=y \frac{\left(m_{2}-1\right)}{a_{2}+x}\left(x-\lambda_{2}\right) \leqslant 0,
$$

and $\lim _{t \rightarrow \infty} y(t)=\eta \geqslant 0 ; \eta>0$ produces a contradiction. The same argument works for $z(t)$ and, with a slight modification, for $x(t)$. This is summarized in the following statement.

Lemma 2.2. If $m_{1} \leqslant 1$ or $\lambda_{1} \geqslant 1$, then $\lim _{t \rightarrow \infty} x(t)=0, \lim _{t \rightarrow \infty} y(t)=0$, $\lim _{t \rightarrow \infty} z(t)=0$. If $m_{2} \leqslant 1$ or $\lambda_{2} \geqslant 1, \lim _{t \rightarrow \infty} y(t)=0$. If $m_{3} \leqslant 1$ or $\lambda_{3} \geqslant 1$, $\lim _{t \rightarrow \infty} z(t)=0$.

We make the following hypothesis for the remainder of the paper

$$
m_{i}>1 \quad \text { and } \quad \lambda_{i}<1, \quad i=1,2,3 ; \quad \lambda_{2}<\lambda_{3} .
$$


The last inequality is merely a choice of labeling for the competing predators and the assumption that they are "different".

\section{A Food Chain}

In this section we consider the special case $z \equiv 0$ where the system (2.1) becomes

$$
\begin{gathered}
S^{\prime}=1-S-\frac{m_{1} x S}{a_{1}+S}, \\
x^{\prime}=\frac{m_{1} x S}{a_{1}+S}-x-\frac{m_{2} x y}{a_{2}+x}, \\
y^{\prime}=\frac{m_{2} x y}{a_{2}+x}-y, \\
S(0)=S_{0} \geqslant 0, \quad x(0)=x_{0} \geqslant 0, \quad y(0)=y_{0} \geqslant 0 .
\end{gathered}
$$

This describes the omega limit set of the system (2.1) when competitive exclusion holds $\left(\lim _{t \rightarrow \infty} z(t)=0\right)$. We describe it first since the results are needed to state the theorem in the next section. It is also a system which is essential for the bifurcation described in Sect. 5. The system (3.1) is of interest in its own right in that it describes a food chain $-y$ eats $x$ which eats $S$. It has been studied in [15], [22], [25] and related experiments are described in [6] and [15]. The previous work has been a local stability analysis and we improve on that in one case, thereby answering a question raised in [22]. Although a local stability analysis exists in the literature we summarize it here because our notation and point of view are slightly different.

This system inherits from the larger system (2.1) the properties that the positive octant is positively invariant, that $m_{1} \leqslant 1$ or $\lambda_{1} \geqslant 1$ forces $\lim _{t \rightarrow \infty} x(t)=0$ (and hence $\lim _{t \rightarrow \infty} y(t)=0$ ), that $m_{2} \leqslant 1$ or $\lambda_{2} \geqslant 1$ forces $\lim _{t \rightarrow \infty} y(t)=0$, and that the omega limit set of any solution of (3.1) lies in the set

$$
\widehat{T}=\{(S, x, y) \mid S+x+y=1, S \geqslant 0, x \geqslant 0, y \geqslant 0\} .
$$

Since every trajectory is asymptotic to its omega limit set, it is sufficient to analyze the system

$$
\begin{aligned}
x^{\prime} & =x\left(\frac{m_{1}(1-x-y)}{1+a_{1}-x-y}-1-\frac{m_{2} y}{a_{2}+x}\right), \\
y^{\prime} & =y\left(\frac{m_{2} x}{a_{2}+x}-1\right)
\end{aligned}
$$

obtained by putting $z=0$ in (2.2).

Note that we may restrict our attention to the triangular region

$$
T=\{(x, y): 0 \leqslant x, y, x+y \leqslant 1\} .
$$

The interesting part of the analysis will be the global stability of the interior critical point of (3.1) but first it is necessary to analyze the local behavior near the critical points on the boundary of $T$. (As noted above this has been done in [3], [4], 
[22].) Critical points on the boundary for (3.1) are

$$
E_{1}=(0,0), \quad E_{2}=\left(1-\lambda_{1}, 0\right)
$$

where $\lambda_{1}=a_{1} /\left(m_{1}-1\right), m_{1}>1$.

The variational matrix takes the form

$$
M=\left[\begin{array}{ll}
m_{11} & m_{12} \\
m_{21} & m_{22}
\end{array}\right],
$$

where

$$
\begin{gathered}
m_{11}=\frac{m_{1}(1-x-y)}{1+a_{1}-x-y}-\frac{m_{2} y}{a_{2}+x}-1+x\left(-\frac{m_{1} a_{1}}{\left(1+a_{1}-x-y\right)^{2}}+\frac{m_{2} y}{\left(a_{2}+x\right)^{2}}\right), \\
m_{12}=\frac{-m_{1} a_{1} x}{\left(1+a_{1}-x-y\right)^{2}}-\frac{m_{2} x}{a_{2}+x}, \\
m_{21}=\frac{m_{2} a_{2} y}{\left(a_{2}+x\right)^{2}}, \quad m_{22}=\frac{m_{2} x}{a_{2}+x}-1 .
\end{gathered}
$$

At $E_{1}$, this takes the form

$$
M=\left[\begin{array}{cr}
\frac{\left(m_{1}-1\right)\left(1-\lambda_{1}\right)}{1+a_{1}} & 0 \\
0 & -1
\end{array}\right]
$$

Thus $E_{1}$ is an asymptotically stable critical point if $m_{1}<1$ or $\lambda_{1}>1$, and a saddle point if $m_{1}>1$ and $\lambda_{1}<1$ (which we are assuming). At $E_{2}$,

$$
M=\left[\begin{array}{cc}
-\left(1-\lambda_{1}\right) \frac{a_{1} m_{1}}{\left(a_{1}+\lambda_{1}\right)^{2}} & -\left(1-\lambda_{1}\right)\left\{\frac{m_{1} a_{1}}{\left(a_{1}+\lambda_{1}\right)^{2}}+\frac{m_{2}}{1+a_{2}-\lambda_{1}}\right\} \\
0 & \frac{\left(m_{2}-1\right)\left(1-\lambda_{1}-\lambda_{2}\right)}{1+a_{2}-\lambda_{1}}
\end{array}\right] .
$$

For $E_{2}$ to be biologically meaningful, i.e., to be in the positive quadrant, it must be the case that $0<\lambda_{1}<1$. Therefore $E_{2}$ is asymptotically stable if $\lambda_{1}+\lambda_{2}>1$ and a saddle point if $0<\lambda_{1}+\lambda_{2}<1$. A stable $E_{2}$ will correspond to extinction of the top level predator and survival of the intermediate level organism.

Suppose now that there is a critical point $\left(x_{c}, y_{c}\right)$ interior to $T$.

$$
\frac{m_{1}\left(1-x_{c}-y_{c}\right)}{1+a_{1}-x_{c}-y_{c}}-\frac{m_{2} y_{c}}{a_{2}+x_{c}}=1, \quad \frac{m_{2} x_{c}}{a_{2}+x_{c}}=1 .
$$

Clearly $x_{c}=\lambda_{2}=a_{2} /\left(m_{2}-1\right)>0$ (if $m_{2}>1$ ), so we are assuming that $y_{c}>0$ satisfies

$$
\frac{m_{1}\left(1-\lambda_{2}-y_{c}\right)}{1+a_{1}-\lambda_{2}-y_{c}}-\frac{m_{2} y_{c}}{a_{2}+\lambda_{2}}=1
$$

Equation (3.3) may be rewritten

$$
\left(m_{1}-1\right)\left(1-\lambda_{2}-\lambda_{1}-y_{c}\right)=\frac{y_{c}}{\lambda_{2}}\left(1+a_{1}-\lambda_{2}-y_{c}\right) .
$$


Since $\lambda_{2}+y_{c}$ must be less than 1 , if $\lambda_{1}+\lambda_{2}>1$ no positive solution of (3.3) exists. Thus if $E_{2}$ is asymptotically stable, there is no interior critical point. If $\lambda_{1}+\lambda_{2}<1$, then (3.3) has precisely one positive solution $y_{c}$.

The variational matrix evaluated at $\left(x_{c}, y_{c}\right)$ takes the form

$$
M=\left[\begin{array}{cc}
\frac{-m_{1} \lambda_{2} a_{1}}{\left(1+a_{1}-\lambda_{2}-y_{c}\right)^{2}}+\frac{m_{2} y_{c} \lambda_{2}}{\left(a_{2}+\lambda_{2}\right)^{2}} & \frac{-m_{1} a_{1} \lambda_{2}}{\left(1+a_{1}-\lambda_{2}-y_{c}\right)^{2}}-1 \\
\frac{\left(m_{2}-1\right) y_{c}}{\lambda_{2}+a_{2}} & 0
\end{array}\right] .
$$

Since the determinant of $M$ is positive, the stability depends upon the trace. The trace of $M$ is negative if

$$
\frac{m_{2} y_{c}}{\left(a_{2}+\lambda_{2}\right)^{2}}<\frac{m_{1} a_{1}}{\left(1+a_{2}-\lambda_{2}-y_{c}\right)^{2}} .
$$

This simplifies to

$$
\frac{y_{c}}{m_{2} \lambda_{2}^{2}}<\frac{m_{1} a_{1}}{\left(1+a_{1}-\lambda_{2}-y_{c}\right)^{2}}
$$

If (3.4) holds, $\left(x_{c}, y_{c}\right)$ is asymptotically stable.

If the inequality is $(3.4)$ is reversed then $\left(x_{c}, y_{c}\right)$ is unstable (a repeller) and by the Poincaré-Bendixson Theorem there is at least one periodic solution. If there is more than one, the inner one is stable from the inside and the outer one is stable from the outside. Two questions immediately arise:

I. If (3.4) is satisfied, is $\left(x_{c}, y_{c}\right)$ globally asymptotically stable?

II. If the inequality in (3.4) is reversed, is the limit cycle unique? (In this case it must be a global attractor w.r.t. non-critical orbits in the open positive $(x, y)$ quadrant)?

Question I which was raised by Sell in [22] is answered the affirmative in Theorem 3.1. It is conjectured that II holds.

In the proof below, the quantity $1-x(t)-y(t)$ turns up frequently, so to save space, we use $S(t)=1-x(t)-y(t)$ for this quantity when convenient. Correspondingly, let $S_{c}=1-x_{c}-y_{c}$. To establish the global stability of the critical point it is only necessary to eliminate limit cycles. Lemma 3.1 is the critical technical step. In the computation necessary to prove the lemma it is convenient to change variables to convert a "time" integral to a line integral in order to be able to apply Green's Theorem. The following observation is useful.

Remark.

$$
S(t)-S_{c}=\frac{\frac{1}{x} \frac{d x}{d t}-\frac{m_{2}}{m_{2}-1}\left(1+\frac{y_{2}}{a_{2}+\lambda_{2}}\right) \frac{1}{y} \frac{d y}{d t}}{\frac{m_{1} a_{1}}{\left(a_{1}+S_{c}\right)\left(a_{1}+S(t)\right)}+\frac{m_{2}}{a_{2}+x}}
$$


Proof of Remark. Since

$$
\begin{aligned}
\frac{x^{\prime}}{x}= & \frac{m_{1} S}{a_{1}+S}-\frac{m_{2} y}{a_{2}+x}-1 \\
= & \frac{m_{1} S}{a_{1}+S}-\frac{m_{1} S_{c}}{a_{1}+S_{c}}+\frac{m_{2} y_{c}}{a_{2}+\lambda_{2}}-\frac{m_{2} y}{a_{2}+x} \\
= & \frac{m_{1} a_{1}\left(S-S_{c}\right)}{\left(a_{1}+S\right)\left(a_{1}+S_{c}\right)}-\frac{m_{2}}{a_{2}+x}\left(y-y_{c}\right)+\frac{m_{2} y_{c}}{\left(a_{2}+\lambda_{2}\right)\left(a_{2}+x\right)}\left(x-x_{c}\right) \\
= & \left(\frac{m_{1} a_{1}}{\left(a_{1}+S\right)\left(a_{1}+S_{c}\right)}+\frac{m_{2}}{a_{2}+x}\right)\left(S-S_{c}\right) \\
& +\left(\frac{m_{2}}{a_{2}+x}+\frac{m_{2} y_{c}}{\left(a_{2}+x\right)\left(a_{2}+\lambda_{2}\right)}\right)\left(x-\lambda_{2}\right) \\
= & \left(\frac{m_{1} a_{1}}{\left(a_{2}+S\right)\left(a_{1}+S_{c}\right)}+\frac{m_{2}}{a_{2}+x}\right)\left(S-S_{c}\right)+\frac{y^{\prime}}{y} \frac{m_{2}}{m_{2}-1}\left(1+\frac{y_{c}}{a_{2}+\lambda_{2}}\right),
\end{aligned}
$$

the remark follows.

For convenience of the statement of the next lemma, rewrite (3.1) as

$$
x^{\prime}=f_{1}(x, y), \quad y^{\prime}=f_{2}(x, y) .
$$

Lemma 3.1. Let $\Gamma(t)=(x(t), y(t))$ be an arbitrary periodic orbit of $(3.1)$ with period $T$. Let $R$ denote the set of points of the plane which are interior to $\Gamma$ and let

$$
\Delta=\int_{0}^{T}\left(\frac{\partial f_{1}}{\partial x}(x(t), y(t))+\frac{\partial f_{2}}{\partial y}(x(t), y(t))\right) d t .
$$

Then

$$
\Delta=\left(\frac{y_{c}}{m_{2} x_{c}}-\frac{m_{1} a_{1} x_{c}}{\left(a_{1}+S_{c}\right)^{2}}\right) T+\iint_{R} Q(x, y) d x d y,
$$

where $Q(x, y)<0$.

Remark. If the constant in (3.6) is negative the orbit $\Gamma$ is asymptotically (orbitally) stable.

Proof of Lemma 3.1. Differentiation yields

$$
\begin{aligned}
\Delta= & \int_{0}^{T}\left\{\left[\frac{m_{1}(1-x-y)}{1+a_{1}-x-y}-\frac{m_{2} y}{a_{2}+x}-1\right]\right. \\
& \left.+x\left[\frac{-m_{1} a_{1}}{\left(1+a_{2}-x-y\right)^{2}}+\frac{m_{2} y}{\left(a_{2}+x\right)^{2}}\right]+\left[\frac{m_{2} x}{a_{2}+x}-1\right]\right\} d t .
\end{aligned}
$$

Since $x(t)$ is periodic of period $T$ the quantity in the first square bracket integrates to zero since it is just $x^{\prime}(t) / x(t)$ and can be integrated as a logarithm. Similarly the third square bracket integrates to zero since it is just $y^{\prime}(t) / y(t)$, where $y(t)$ is periodic of 
period $T$. Thus

$$
\Delta=\int_{0}^{T}\left(\frac{m_{2} y(t)}{\left(a_{2}+x(t)\right)^{2}}-\frac{a_{1} m_{1}}{\left(1+a_{1}-x(t)-y(t)\right)^{2}}\right) x(t) d t .
$$

The rest of the proof is simply a restructuring of this integral so that an application of Green's Theorem yields a function $Q(x, y)$ with the desired sign. The details of this are somewhat complicated and tedious.

First of all

$$
\begin{aligned}
\int_{0}^{T} \frac{m_{2} x(t) y(t)}{\left(a_{2}+x(t)\right)^{2}} d t= & \int_{0}^{T}\left(\frac{x(t)}{a_{2}+x(t)}\right)\left(\frac{m_{2} y(t)}{a_{2}+x(t)}\right) d t \\
= & \int_{0}^{T} \frac{x(t)}{a_{2}+x(t)}\left(\frac{m_{1} S(t)}{a_{1}+S(t)}-1-\frac{x^{\prime}(t)}{x(t)}\right) d t \\
= & \int_{0}^{T} \frac{x(t)}{a_{2}+x(t)}\left(\frac{m_{1} S_{c}}{a_{1}+S_{c}}-1\right) d t \\
& +\int_{0}^{T} \frac{x(t)}{a_{2}+x(t)}\left(\frac{m_{1} S(t)}{a_{2}+x(t)}-\frac{m_{1} S_{c}}{a_{1}+S_{c}}\right) d t \\
= & I_{1}+I_{2} .
\end{aligned}
$$

We begin with $I_{2}$. Combining terms yields

$$
\begin{aligned}
I_{2} & =\int_{0}^{T} \frac{x(t)}{a_{2}+x(t)}\left(\frac{m_{1} S(t)\left(a_{1}+S_{c}\right)-m_{1} S_{c}\left(a_{1}+S(t)\right)}{\left(a_{1}+S(t)\right)\left(a_{1}+S_{c}\right)}\right) d t \\
& =\frac{m_{1} a_{1}}{a_{1}+S_{c}} \int_{0}^{T}\left(\frac{x(t)}{a_{2}+x(t)} \frac{S(t)-S_{c}}{a_{1}+S(t)}\right) d t .
\end{aligned}
$$

An application of the remark preceding the lemma yields that

$$
\begin{aligned}
I_{2}= & \frac{m_{1} a_{1}}{a_{1}+S_{c}} \int_{\Gamma} \frac{x}{\left(a_{2}+x\right)\left(a_{1}+S\right)}\left\{\frac{1}{\left(\frac{m_{1} a_{1}}{\left(a_{1}+S_{c}\right)\left(a_{2}+S\right)}+\frac{m_{2}}{a_{2}+x}\right)} \frac{d x}{x}\right. \\
& \left.-\frac{m_{2}}{m_{2}-1}\left(1+\frac{y_{c}}{a_{2}+\lambda_{2}}\right) \frac{d y}{y}\right\} .
\end{aligned}
$$

Green's Theorem is now applied to give

$$
I_{2}=\frac{m_{1} a_{1}}{a_{1}+S_{c}} \iint_{R}\left(\frac{\partial Q_{1}}{\partial x}-\frac{\hat{\partial} P_{1}}{\partial y}\right) d x d y
$$

where

$$
\begin{aligned}
& Q_{1}(x, y)=-\frac{m_{2}}{m_{2}-1}\left(1+\frac{y_{c}}{m_{2} \lambda_{2}}\right) \frac{x}{y} P(x, y), \\
& P_{1}(x, y)=\left(a_{2}+x\right)^{-1} P(x, y)
\end{aligned}
$$




$$
P(x, y)=\frac{1}{\frac{m_{1} a_{1}}{a_{1}+S_{c}}+\frac{m_{2}\left(a_{1}+1-x-y\right)}{a_{2}+x}} .
$$

It follows, upon differentiating, that

$$
\frac{\partial Q_{1}}{\partial x}<0, \quad \frac{\partial P_{1}}{\partial y}>0
$$

and the integrand is negative. We return now to $I_{1}$. Since

$$
I_{1}=\left(\frac{m_{1} S_{c}}{a_{1}+S_{c}}-1\right) \int_{0}^{T} \frac{x(t)}{a_{2}+x(t)} d t
$$

then, using the differential equation for $y$, one has

$$
I_{1}=\left(\frac{1}{m_{2}} \cdot \frac{m_{1} S_{c}}{a_{1}+S_{c}}-1\right) \int_{0}^{T}\left(\frac{y^{\prime}}{y}+1\right) d t=\frac{T}{m_{2}}\left(\frac{m_{1} S_{c}}{a_{1}+S_{c}}-1\right)=T \frac{y_{c}}{a_{2}+\lambda_{2}}=\frac{y_{c} T}{m_{2} \lambda_{2}} \text {. }
$$

We return now to the second integral in (3.7) and apply the same technique. Write

$$
\begin{aligned}
-\int_{0}^{T} \frac{a_{1} m_{1} x(t)}{\left(a_{1}+S(t)\right)^{2}} d t= & -\int_{0}^{T} \frac{x(t)}{a_{2}+x(t)}\left\{\left[\frac{m_{1} a_{1}\left(a_{2}+x\right)}{\left(a_{1}+S(t)\right)^{2}}-\frac{m_{1} a_{1}}{\left(a_{1}+S_{c}\right)^{2}}\left(a_{2}+x\right)\right]\right. \\
& +\left[\frac{m_{1} a_{1}\left(a_{2}+x\right)}{\left(a_{1}+S_{c}\right)^{2}}-\frac{m_{1} a_{1}\left(a_{2}+\lambda_{2}\right)}{\left(a_{1}+S_{c}\right)^{2}}\right] \\
& \left.+\left[\frac{m_{1} a_{1}\left(a_{2}+\lambda_{2}\right)}{\left(a_{1}+S_{c}\right)^{2}}\right]\right\} d t \\
= & I_{3}+I_{4}+I_{5} .
\end{aligned}
$$

First of all

$$
\begin{aligned}
I_{3} & =-\int_{0}^{T} \frac{x}{a_{2}+x}\left[\frac{m_{1} a_{1}\left(a_{2}+x\right)}{\left(a_{1}+S\right)^{2}}-\frac{m_{1} a_{1}\left(a_{2}+x\right)}{\left(a_{1}+S_{c}\right)^{2}}\right] d t \\
& =a_{1} m_{1} \int_{0}^{T} \frac{x\left(S-S_{c}\right)\left(2 a_{1}+S+S_{c}\right)}{\left(a_{1}+S_{c}\right)^{2}\left(a_{1}+S\right)^{2}} d t
\end{aligned}
$$

which can be broken into two integrals as

$$
I_{3}=a_{1} m_{1} \frac{1}{\left(a_{1}+S_{c}\right)^{2}} \int_{0}^{T} \frac{x\left(S-S_{c}\right)}{a_{1}+S} d t+\frac{1}{a_{1}+S_{c}} \int_{0}^{T} \frac{x\left(S-S_{c}\right)}{\left(a_{1}+S\right)^{2}} d t
$$

each of which can be converted to a line integral using the remark preceding the lemma. We omit the details but the end result is as follows:

$$
\int_{0}^{T} \frac{x\left(S-S_{c}\right)}{a_{1}+S} d t=\iint_{R}\left(\frac{\partial Q_{2}}{\partial x}-\frac{\partial P_{2}}{\partial y}\right) d x d y
$$

where

$$
P_{2}=P(x, y), \quad Q_{2}=-\frac{m_{2}}{m_{2}-1}\left(1+\frac{y_{c}}{m_{2} \lambda_{2}}\right) \frac{x}{y} P(x, y)
$$


and

$$
\begin{aligned}
& \int_{0}^{T} \frac{x\left(S-S_{c}\right)}{\left(a_{1}+S\right)^{2}} d t=\iint_{R}\left(\frac{\partial Q_{3}}{\partial x}-\frac{\partial P_{3}}{\partial y}\right) d x d y, \\
& P_{3}=\frac{P(x, y)}{1+a_{1}-x-y}, \\
& Q_{3}=\frac{m_{2}}{m_{2}-1}\left(1+\frac{y_{c}}{m_{2} \lambda_{2}} \frac{x P(x, y)}{y\left(1+a_{1}-x-y\right)}\right) .
\end{aligned}
$$

Furthermore $\partial Q_{2} / \partial x<0, \partial Q_{3} / \partial x<0, \partial P_{2} / \partial y>0, \partial P_{3} / \partial y>0$. Thus $I_{3}$ can be written as an integral over $R$ with a negative integrand.

Finally

$$
I_{4}=-\frac{m_{1} a_{1}}{\left(m_{2}-1\right)\left(a_{1}+S_{c}\right)^{2}} \int_{\Gamma} \frac{x}{y} d y=-\frac{m_{1} a_{1}}{\left(m_{2}-1\right)\left(a_{1}+S_{c}\right)^{2}} \iint_{R} \frac{1}{y} d x d y
$$

and

$$
I_{5}=-\frac{m_{1} a_{1}\left(a_{2}+\lambda_{2}\right)}{m_{2}\left(a_{1}+S_{c}\right)^{2}} \int_{0}^{T}\left(\frac{y^{\prime}}{y}+1\right) d t=\frac{-m_{1} a_{1}\left(a_{2}+\lambda_{2}\right)}{m_{2}\left(a_{1}+S_{c}\right)^{2}} T=\frac{-m_{1} a_{1} \lambda_{2}}{\left(a_{1}+S_{c}\right)^{2}} T .
$$

Adding all of the above integrals yields

$$
\Delta=\left(\frac{-m_{1} a_{1} \lambda_{2}}{\left(a_{1}+S_{c}\right)^{2}}+\frac{y_{c}}{m_{2} \lambda_{2}}\right) T+\iint_{R} Q(x, y) d x d y
$$

where $Q(x, y)<0$, and the proof of the lemma is complete.

Theorem 3.1. If the critical point $\left(x_{c}, y_{c}\right)$ satisfies

$$
\frac{y_{c}}{m_{2} \lambda_{2}^{2}}<\frac{m_{1} a_{1}}{\left(1+a_{1}-\lambda_{2}-y_{c}\right)^{2}}
$$

then $\left(x_{c}, y_{c}\right)$ is globally asymptotically stable w.r.t. the interior of the first quadrant.

Proof. The condition (3.4) is just the condition for the local asymptotic stability of $\left(x_{c}, y_{c}\right)$ as determined by the linearization. Suppose $P$ is an arbitrary periodic orbit about $\left(x_{c}, y_{c}\right)$. Then by the remark following the statement of Lemma 3.1, every such orbit is asymptotically stable-a contradiction since an asymptotically stable critical point mandates at least one periodic orbit being unstable. Thus there are no periodic orbits and the local asymptotic stability of $\left(x_{c}, y_{c}\right)$ is global by the PoincaréBendixson Theorem and by the lack of connected saddle points.

Theorem 3.2. If $\left(x_{c}, y_{c}\right)$ exists in the positive octant and

$$
\frac{y_{c}}{m_{2} \lambda_{2}^{2}}>\frac{m_{1} a_{1}}{\left(1+a_{1}-\lambda_{2}-y_{c}\right)^{2}}
$$

then there exists a periodic orbit for (3.3).

Proof. The proof (as noted above and in [22]) follows from the Poincaré-Bendixson Theorem. 


\section{Competitive Exclusion}

In this section we obtain conditions that guarantee that $\lim _{t \rightarrow \infty} z(t)=0$, that is, one of the predators becomes extinct. Because of $(\mathrm{H}-1)$ the system is prejudiced in favor of $y(t)$; symmetric results could be stated for the conclusion $\lim _{t \rightarrow \infty} y(t)=0$ if $(\mathrm{H}-1)$ is not assumed. Two lemmas are presented which guarantee that the omega limit set of any solution of $(2,1)$ has $z$ coordinate equal to zero. The first lemma is quite easy to prove and we state it separately even though it could be included in Lemma 4.2. It is a standard observation for chemostat equations [7, Fig. 3].

Lemma 4.1. Let $(\mathrm{H}-1)$ hold and let $f_{2}(x)=m_{2} /\left(a_{2}+x\right)$ and $f_{3}(x)=m_{3} /\left(a_{3}+x\right)$. If $f_{2}(x)>f_{3}(x), 0 \leqslant x \leqslant 1$, then $\lim _{t \rightarrow \infty} z(t)=0$ for every solution of $(2.1)$ with positive initial conditions.

Proof. Let $(S(t), x(t), y(t), z(t))$ be a solution of (2.1). Then

$$
\begin{aligned}
\frac{z^{\prime}(t)}{z(t)}-\frac{y^{\prime}(t)}{y(t)} & =x(t)\left[f_{3}(x)-f_{2}(x)\right] \\
& \leqslant\left(\inf _{t} x(t)\right)\left(\delta_{1}\right)
\end{aligned}
$$

where $\delta_{1}=\max _{0 \leqslant x \leqslant 1}\left[f_{3}(x)-f_{2}(x)\right]<0$. If inf $x(t)=0$, then an easy limit argument shows $\lim _{t \rightarrow \infty} z(t)=0$ (and $\lim _{t \rightarrow \infty} y(t)=0$ ). If $\inf _{t} x(t)=\delta_{2}>0$, then let $\delta=\delta_{1} \delta_{2}$ so that

$$
\frac{z^{\prime}(t)}{z(t)}-\frac{y^{\prime}(t)}{y(t)} \leqslant \delta<0 .
$$

Then

$$
z(t) \leqslant c y(t) e^{-\delta t}
$$

for some $c>0$. Since $y(t)$ is bounded, $\lim _{t \rightarrow \infty} z(t)=0$.

This lemma has a simple biological interpretation; if $y$ is a better competitor (has a higher intrinsic growth rate) for every level of the prey $x$, then $z$ will become extinct. This would be true for example if $m_{2} \geqslant m_{3}$ and $a_{2} \leqslant a_{3}$ and at least one of the inequalities is strict. If there is a value $x^{*} \in(0,1)$ such that $f_{2}\left(x^{*}\right)=f_{3}\left(x^{*}\right)$, and the two curves, $w=f_{i}(x), i=2,3$, cross, then $y$ has a higher growth rate for $x$ on one side of $x^{*}$ and $z$, for $x$ on the other. Coexistence then is conceivable if $x(t)$ oscillates about $x^{*}$. The next lemma rules out one such case.

Lemma 4.2. Let $(\mathrm{H}-1)$ hold. If $m_{3} \leqslant m_{2}$, then $\lim _{t \rightarrow \infty} z(t)=0$.

Proof. The proof of this lemma requires a fairly lengthy computation and the reader is referred to Lemma 4.3 of [11] for the proof of a quite similar lemma. Lemma 4.2 follows in the same manner.

The biological basis of the proof is quite easy to see. Although the curves cross at $x^{*}, 0<\lambda_{2}<\lambda_{3}<1$ forces $x^{*}<\lambda_{2}$. Thus at the prey level where $z$ has an advantage both predators are decreasing exponentially; when the prey recovers (reaches a higher level) $y$ has the advantage. 
The following theorem follows from Lemma 4.2 and Theorems 3.2 and 3.3.

Theorem 4.1. Let $m_{3} \leqslant m_{2}$ and let $(\mathrm{H}-1)$ hold. If $(S(t), x(t), y(t), z(t))$ is a solution of (2.1) with positive initial conditions, then

$$
\lim _{t \rightarrow \infty}(S(t), x(t), y(t), z(t))=\left(1-x_{c}-y_{c}, x_{c}, y_{c}, 0\right)
$$

if

$$
\frac{y_{c}}{m_{2} x_{c}^{2}}<\frac{m_{1} a_{1}}{\left(1+a_{1}-x_{c}-y_{c}\right)^{2}},
$$

where $x_{c}=\lambda_{2}$ and $y_{c}$ is a positive solution of

$$
\frac{m_{1}\left(1-\lambda_{2}-y_{c}\right)}{1+a_{1}-\lambda_{2}-y_{c}}-\frac{m_{2} y}{a_{2}+\lambda_{2}}=1 .
$$

If the inequality (4.1) is reversed and $(S(t), x(t), y(t), z(t))$ is a noncritical orbit, then it approaches (or is) a periodic orbit in the plane $S+x+y=1, z=0$.

Since (H-1) forces $m_{3}<m_{2}$ if $a_{3} \leqslant a_{2}$, the only case where coexistence is possible is $a_{2}<a_{3}, m_{2}<m_{3}$.

\section{Coexistence}

In Sect. 3 it was shown that the system (2.1) possesses a limit cycle in the plane $S+x+y=1, z=0$, provided that

$$
\frac{y_{c}}{m_{2} x_{c}^{2}}>\frac{m_{1} a_{1}}{\left(1+a_{1}-x_{c}-y_{c}\right)^{2}} .
$$

The uniqueness of the limit cycle and its stability properties were not established, in contrast to the case of a similar problem with a logistically growing prey [5]. If more than one limit cycle should exist then the innermost one is stable from the inside and the outer one is stable from the outside, so for these two, one Floquet multiplier is one and the remaining Floquet multiplier cannot be larger than one. In fact, from [7] it would follow that there is at least one stable limit cycle. We need to make a slightly stronger assumption:

(H-2) There exists a limit cycle for (3.1) which has a Floquet multiplier (strictly) inside the unit circle.

With this assumption, we can show that there is a range in the parameter space where competitive exclusion does not hold. The uniqueness proof given in [5] was strong enough to yield $(\mathrm{H}-2)$ as well, but symmetries used there are not present in (3.1).

Theorem 5.1. Let $a_{i}, m_{i}, i=1,2$, be fixed so that $m_{i}>1, \lambda_{i}<1$ and $(\mathrm{H}-2)$ holds. Fix $m_{3}>m_{2}$. Then there exists a number $a_{3}^{*}>a_{2}$ such that for $a_{3}<a_{3}^{*},\left|a_{3}-a_{3}^{*}\right|$ sufficiently small, we have $\lambda_{2}<\lambda_{3}$ and (2.2) has a periodic orbit in the positive octant which is arbitrarily near the $x-y$ plane. $((2.1)$ has a periodic orbit in the positive cone in $E^{4}$ arbitrarily near the plane, $\left.S+x+y=1, z=0\right)$. 
The proof requires some preliminary notation and auxiliary lemmas.

Let $x(t), y(t)$ be a periodic solution of period $T$ of the system (3.1) which is given by (H-2) for the parameter values $m_{i}, a_{i}, i=1,2$. Then $(x(t), y(t), 0)$ is a solution of (2.2) for any choice of $m_{3}$ and $a_{3}$. Fix $m_{3}$ and define

$$
\mu\left(a_{3}\right)=\frac{m_{3}}{T} \int_{0}^{T} \frac{x(\xi)}{a_{3}+x(\xi)} d \xi
$$

$a_{3}$ will be treated as a bifurcation parameter.

Let $\Gamma$ be the orbit corresponding to this periodic solution. For each value of $a_{3}$ the Poincare map associated with $\Gamma, P: W_{0} \rightarrow W_{1}$ exists, where $W_{0}, W_{1}$ are open subsets of $\Omega$, a two-dimensional, transverse section of $\Gamma$. The linearization about the periodic orbit and the linearization of the Poincaré map about the fixed point corresponding to $\Gamma$ are related. The following statement makes this point precise.

Lemma 5.1 [18]. The spectrum of the linearization of the Poincare map union $\{1\}$ is equal to the spectrum of the linearization of the solution map defined by

$$
\Phi\left(x^{0}, y^{0}, z^{0}\right)=(x(T), y(T), z(T)),
$$

where

$$
x(0)=x^{0}, \quad y(0)=y^{0}, \quad z(0)=z^{0} .
$$

As a consequence of Lemma 5.1, showing that one Floquet multiplier passes from inside to outside the unit circle shows that an eigenvalue of the linearization of the Poincaré map passes from inside to outside the unit circle. This is the key ingredient in the following bifurcation theorem [18, p. 24].

Lemma 5.2. Let $W$ be an open neighborhood of $O \in R^{2}$ and let $I$ be an open interval about $O \in R$. Let $\Phi_{v}: W \rightarrow R^{n}$ be such that the map $(v, x) \rightarrow \Phi_{v}(x)$ is a $C^{k}$ map $(k \geqslant 1)$ from $I \times W$ to $R^{2}$, and such that $\Phi_{v}(0)=0$ for all $v \in I$. Define $L_{v}$ to be the differential map $d \Phi_{v}(0)$ and suppose that all eigenvalues of $L_{v}$ lie inside the unit circle of the complex plane for $v<0$. Assume that there is a real, simple eigenvalue $l(v)$ of $L_{v}$ such that $l(0)=1$ and $(d l / d v)(0)>0$. Let $v_{0}$ be the eigenvector corresponding to $l(0)$. Then there is a $C^{k-1}$ curve $\mathscr{C}$ of fixed points of $\Phi:(v, x) \rightarrow\left(v, \Phi_{v}(x)\right)$ near $(0,0)$ in $I \times R^{2}$ which together with the points $(v, 0)$ are the only fixed points of $\Phi$ near $(0,0)$. The curve $\mathscr{C}$ is tangent to $v_{0}$ at $(0,0)$ in $I \times R^{2}$.

Remark. The direction of tangency of $\mathscr{C}$ given in the last assertion of the lemma is more specific than that given in [18] but is easily obtained from the proof. We require this more precise location of $\mathscr{C}$ in our application. The following lemma from [2] follows from elementary differential equations theory (see also [23]).

Lemma 5.3. Let $A(t)$ be $a 2 \times 2$ periodic matrix of period $\tau$ and suppose that the linear system

$$
\left(\begin{array}{l}
y_{1} \\
y_{2}
\end{array}\right)^{\prime}=A(t)\left(\begin{array}{l}
y_{1} \\
y_{2}
\end{array}\right)
$$

has Floquet exponents 0 and $-\gamma<0$. Let $b(t), c(t)$ be functions of period $\tau$ such that the mean value of $b$ is equal to $\eta<0$. Then the linear system 


$$
\left(\begin{array}{l}
y_{1} \\
y_{2} \\
y_{3}
\end{array}\right)^{\prime}=B(t)\left(\begin{array}{l}
y_{1} \\
y_{2} \\
y_{3}
\end{array}\right)
$$

where

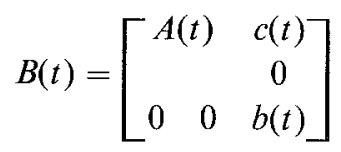

has Floquet exponents $0,-\gamma, \eta$.

Proof of Theorem 5.1. Let $a_{3}^{*}$ be a constant to be determined, and let $v=a_{3}^{*}-a_{3}$. Let $\Gamma$ be the orbit associated with the periodic solution $(x(t), y(t), 0)$ when $a_{3}=a_{3}^{*}$ $(v=0)$. Fix a point $p_{0} \in \Gamma$ and let $\Omega$ be the transverse section to $\Gamma$ at $p_{0}$, identifying $p_{0}$ with 0 in $E^{2}$. Let $\Phi_{v}$ denote the Poincare map associated with the periodic solution $(x(t), y(t), 0)$ for $v$ near 0 . There is a neighborhood $W_{0}$ of $p_{0}$ in $\Omega$ such that for $v$ sufficiently close to zero, $\Phi_{v}$ is defined on $W_{0}$. We investigate the spectrum of $L_{v}=d \Phi_{v}(0)$ by examining the Floquet multipliers of the corresponding system of differential equations.

The matrix associated with the linearization of (2.2) takes the form

$$
\left[\begin{array}{ccc}
f_{x}(x(t), y(t), 0) & f_{y}(x(t), y(t), 0) & f_{z}(x(t), y(t), 0) \\
y(t) \frac{m_{2} a_{2}}{\left(a_{2}+x(t)\right)^{2}} & \frac{m_{2} x(t)}{a_{2}+x(t)}-1 & 0 \\
z(t) \frac{m_{3} a_{3}}{\left(a_{2}+x(t)\right)^{2}} & 0 & \frac{m_{3} x(t)}{a_{3}+x(t)}-1
\end{array}\right]
$$

where $x^{\prime}=f(x, y, z)$ in (2.2).

This can be viewed as partitioned in the form

$$
M=\left[\begin{array}{ccc}
V & f_{z}(x(t), y(t), 0) \\
& & 0 \\
0 & 0 & \frac{m_{3} x(t)}{a_{3}+x(t)}-1
\end{array}\right],
$$

where the two-dimensional system

$$
\check{\zeta}^{\prime}=V(t) \xi
$$

has one Floquet multiplier equal to $1\left(\right.$ since $\xi(t)=\left(\begin{array}{l}x(t) \\ y(t)\end{array}\right)$ is a periodic solution) and one multiplier (call it $e^{-\gamma}$ ) inside the unit circle, by the hypothesis (H-2) on the planar orbit. Thus the linearization of the Poincare map has eigenvalues $e^{-\gamma}, e^{r}$ where

$$
r=\mu\left(a_{3}\right)-1
$$

Now

$$
\mu(0)=m_{3}>1
$$


by hypothesis and

$$
\mu\left(a_{3}\right) \leqslant \frac{m_{3}}{a_{3} T} \int_{0}^{T} x(\xi) d \xi
$$

so for $a_{3}$ sufficiently large, $\mu\left(a_{3}\right)$ is arbitrarily small (note that $x(t)$ is independent of $\left.a_{3}\right) . \mu\left(a_{3}\right)$ is continuous and decreasing with $a_{3}$, so there is a unique value $a_{3}^{*}$ of $a_{3}$ such that $\mu\left(a_{3}^{*}\right)=1$. Note also that

$$
\frac{d \mu}{d a_{3}}=\frac{-m_{3}}{T} \int_{0}^{T} \frac{x(\xi)}{\left(a_{3}+x(\xi)\right)^{2}} d \xi<0 .
$$

In particular, $\mu\left(a_{3}\right)$ crosses the value 1 non tangentially as $a_{3}$ passes through $a_{3}^{*}$ (as $r$ passes through 0 ) so the Floquet multiplier passes through the unit circle transversally; indeed

$$
\frac{\partial e^{r}}{\partial a_{3}}\left(a_{3}^{*}\right)=\frac{\partial \mu}{\partial a_{3}}\left(a_{3}^{*}\right) \cdot \exp \left[\mu\left(a_{3}^{*}-1\right)\right]<0
$$

as $r$ passes through zero. Thus $\left(\partial e^{r} / \partial v\right)(0)>0$.

The same is now true for the linearization of the Poincare map $\Phi_{r}-$ i.e., $L_{r}$ has eigenvalues $e^{-\gamma}, e^{r}$ and $e^{r}$ crosses the unit circle nontangentially as $r$ passes through zero.

Applying Lemma 5.2 there is a $C^{1}$ curve $\mathscr{C}$, of fixed points of $\Phi_{v}(x)$, bifurcating from $(v, 0)$ at $(0,0)$. Each such fixed point corresponds to a periodic solution of $(2.2)$ and hence of (2.1). Since $\mathscr{C}$ is tangent to the eigenvector associated with the eigenvalue 1 of $L_{0}$, it is transverse to the $x$ - $y$ plane. Hence one branch lies in the positive octant if $\left|a_{3}-a_{3}^{*}\right|$ is sufficiently small. Since

$$
\mu\left(a_{2}\right)=\frac{m_{3}}{T} \int_{0}^{T} \frac{x(\xi)}{a_{2}+x(\xi)} d \xi>\frac{m_{2}}{T} \int_{0}^{T} \frac{x(\xi)}{a_{2}+x(\xi)} d \xi=1,
$$

$a_{3}^{*}>a_{2}$, so $a_{3}$ may be chosen to satisfy $a_{3}>a_{2}$.

It remains to show that $\lambda_{2}<\lambda_{3}$ by the choice $a_{3}<a_{3}^{*}$. Note that the choice $\bar{a}_{3}=a_{2}\left(m_{3}-1\right) /\left(m_{2}-1\right)$ produces $\lambda_{2}=\lambda_{3}$, so $a_{3}>\bar{a}_{3}$ will imply $\lambda_{2}<\lambda_{3}$. Suppose that $a_{3}^{*} \leqslant \bar{a}_{3}$. Let $a_{3}$ be chosen such that $a_{2}<a_{3}<a_{3}^{*}$, and sufficiently close to $a_{3}^{*}$ that there is a periodic solution $(\tilde{x}(t), \tilde{y}(t), \tilde{z}(t))$ of $(2.2)$, of period $\tilde{T}$, guaranteed by the bifurcation at $a_{3}^{*}$. Thus since $a_{2}<a_{3}<\bar{a}_{3}$, we have

$$
1=\mu\left(a_{2}\right)>\mu\left(a_{3}\right)>\mu\left(\bar{a}_{3}\right) \text {. }
$$

Hence,

$$
\tilde{z}(n \tilde{T})=\tilde{z}(0) \exp \left[n \tilde{T}\left(\mu\left(a_{3}\right)-1\right)\right]
$$

or

$$
\lim _{n \rightarrow \infty} \tilde{z}(n \tilde{T})=0
$$

contradicting the periodicity of the solution. Hence $a_{3}^{*}>\bar{a}_{3}$. Thus for $a_{3}<a_{3}^{*}$ and sufficiently close, we shall have $a_{3}>\bar{a}_{3}$ and so $\lambda_{2}<\lambda_{3}$. This completes the proof of the theorem. The limit cycle which bifurcates into the positive octant represents two 
coexisting predators, $y, z$ feeding on a single prey $x$ (which of course is supported by the nutrient $S$ ).

\section{Some Comments on Genericity}

It is customary to say that a property $P$ is generic for the system of differential equations.

$$
x^{\prime}=f(x), \quad x \in R^{n}, \quad f: R^{n} \rightarrow R^{n}
$$

if $P$ holds for $f$ belonging to a residual set (having 1 st category complement) in an appropriate function space. The idea is that the right-hand side of (6.1) is an "approximation" to the "true" situation and so, to be "real", a property should be preserved for "most" small perturbations of $f$. The property of invariant faces of the positive cone for system (2.1) can be destroyed by "most" small perturbations of $f$. Moreover the "generic" bifurcation of a limit cycle is that of bifurcation into an invariant torus - why does it not occur for (2.2)? This section addresses this question.

The model for a self-renewing organism is usually made by prescribing the intrinsic growth rate - the per capita growth rate. For example a population growing logistically is modeled by setting the intrinsic growth rate equal to a linear function

$$
\frac{x^{\prime}}{x}=1-\frac{x}{K}
$$

or, more generally, for a self-renewing organism is modeled by

$$
\frac{x^{\prime}}{x}=f(x), \quad x \in \mathbf{R}
$$

where the choice of $f(x)$ specifies the model. In keeping with the spirit of the idea of generic, it is the $f(x)$ which is "approximate", not $x f(x)$. With this view, the invariant faces of the positive cone of a system

$$
x_{i}^{\prime}=x_{i} f\left(x_{1} \cdots x_{n}\right)
$$

are perfectly "generic". They are preserved if $f$ is perturbed, for $x_{i}=0$ remains a solution. This corresponds, of course, to the biological principle that from zero organisms, none are produced. Three of the equations in (2.1) are of this form and the remaining one is of that form plus a constant positive forcing term, corresponding to the nutrient input (which of course is not self-generating). After the transformation to (2.2) all are of the form of the above since the appropriate zero solution is maintained under perturbations.

The second question is "why not an invariant torus?" The answer is that a limit cycle is an invariant plane can only be perturbed into another limit cycle. We restrict ourselves to three dimensions, as in (2.2) but there is a general principle here that after bifurcation from an invariant face, the structure is not more complicated.

Consider the system

$$
x^{\prime}=x f_{1}(x, y, z), \quad y^{\prime}=y f_{2}(x, y, z), \quad z^{\prime}=z f_{3}(x, y, z)
$$


and suppose that $(x(t), y(t), 0)$ is a limit cycle in the $z=0$ face. $(x(t), y(t))$, of course, is also a periodic solution of

$$
y^{\prime}=x f_{1}(x, y, 0), \quad y^{\prime}=y f_{2}(x, y, 0) .
$$

The variational equation corresponding to linearization of (6.4) about the periodic solution has two Floquet exponents - one of which is zero because of the periodic solution. Thus the other must be real-they are eigenvalues of a real matrix. The variational matrix for (6.3) about this same solution contains the variational matrix for (6.4) in the upper left-hand corner and necessarily has only one entry in the third row, in the 3,3 position. Thus the above two real Floquet exponents are exponents of the larger system, and the third exponent is necessarily real. (We note that we are taking some liberty with the terminology since it is multipliers and not exponents which are uniquely defined.)

The eigenvalues of the Poincaré map corresponding to the Floquet multipliers, may pass through the unit circle, through a change in parameters, by passing through +1 or -1 or with nontrivial complex part. Since the multipliers are real, the last case - which is needed for bifurcation into an invariant torus - is thus impossible. Passage through -1 which produces period doubling is also not possible since the exponents are real. Passage through +1 corresponds to the kind of bifurcation shown for (2.2).

Finally it should be noted that after bifurcation out of the plane, secondary bifurcations may be of any type. Invariant tori and periodic doubling bifurcations are not prohibited in the system, it is only that they cannot arise from a bifurcation out of a limit cycle in an invariant bounding plane.

\section{References}

1. Butler, G. J.: Coexistence in predator-prey systems. In: Burton, T. (ed.) Modeling and differential equations in biology. New York: Marcel Dekker 1980

2. Butler, G. J., Waltman, P.: Bifurcation from a limit cycle in a two predator-one prey ecosystem modeled on a chemostat. J. Math. Biology 12, 295-310 (1981)

3. Canale, R. P.: Prey relationships in a model for activated process. Biotechnol. Bioengineering 11, $887-907$ (1969)

4. Canale, R. P.: An analysis of models describing predator prey interaction. Biotechnol. Bioengineering 12, 353-378 (1970)

5. Cheng, K. S.: Uniqueness of limit cycle for a predator-prey system. SIAM J. Math. Analysis 12, $541-548(1981)$

6. Drake, J. F., Tsuchiya, H. M.: Predation of Escherichia Coli by Colpoda Stenii. Appl. Envr. Microbiol. 31, 870-874 (1976)

7. Erle, D. : Stable closed orbits in plane autonomous dynamical systems. J. Reine Angew. Math. 305, $136-139$ (1979)

8. Fredrickson, A. G., Stephanopoulos, G.: Microbial competition. Science 213, $972-979$ (1981)

9. Hansen, S. R., Hubbell, S. P.: Single-nutrient microbial competition: Agreement between experimental and theoretical forecast outcomes. Science 207, $1491-1493$ (1980)

10. Herbert, D., Elsworth, R., Telling, R. C.: The continuous culture of bacteria: A theoretical and experimental study. J. Gen. Microbiol. 14, 601-622 (1956)

11. Hsu, S. B., Hubbell, S., Waltman, P.: A mathematical theory for single-nutrient competition in continuous cultures of microorganisms. SIAM J. Appl. Math. 32, 366-383 (1977)

12. Hsu, S. B., Hubbell, S. P., Waltman, P.: A contribution to the theory of competing predators. Ecol. Mongr. 48, $337-349$ (1978)

13. Hsu, S. B.: Limiting behavior for competing species. SIAM J. Appl. Math. 34, 760-763 (1978) 
14. Jannash, H. W., Mateles, R. T.: Experimental bacterial ecology studied in continuous culture. Adv. Microb. Physiol. 11, $165-212$ (1974)

15. Jost, J. L., Drake, S. F., Fredrickson, A. G., Tsuchiya, M.: Interaction of tetrahymena pyriformis, escherichia coli, azotobacter vinelandii and glucose in a minimal medium. J. Bacteriol. 113, $834-840(1976)$

16. Keener, J. P.: Oscillatory coexistence in the chemostat: A codimension two unfolding, SIAM J. Appl. Math. (to appear)

17. Koch, A. L.: Competitive coexistence of two predators utilizing the same prey under constant environmental conditions. J. Theoret. Biology 44, $378-386$ (1974)

18. Marsden, J. E., McCracken, M. : The Hopf bifurcation and its applications. Berlin-Heidelberg-New York: Springer 1976

19. McGehee, R., Armstrong, R. A.: Some mathematical problems concerning the ecological principle of competitive exclusion. J. Diff. Eq. 23, 30-52 (1977)

20. Monod, J.: Recherches sur la croissance des cultures bacteriennes. Paris: Hermann 1942

21. Pike, E. B., Cuids, C. R.: The microbial ecology of activated sludge process. In: Sykes, G., Skinner, F. A. (eds) Microbial aspects of pollution. New York: Academic Press 1971

22. Sell, G.: What is a dynamical system? In: Hale, J. (ed) Studies in ordinary differential equations. MAA Studies in Mathematics No. 14, 1977

23. Smith, H. L.: The interaction of steady state and Hopf bifurcation in a two-predator-one-prey competition model. SIAM J. Appl. Math. 42, 27-43 (1982)

24. Stewart, F. M., Levin, B. R.: Partitioning of resources and the outcome of interspecific competition; A model and some general considerations. Am. Nat. 107, 171-198 (1973)

25. Tsuchiya, H. M., Drake, S. F., Jost, J. L., Fredrickson, A. G.: Predator-prey interactions of dictyostelium discordeum and escherichia coli in continuous culture. J. Bacteriol. 110, 1147-1153 (1972)

26. Veldcamp, H. : Ecological studies with the chemostat. Adv. Microbial Ecol. 1, 59 - 95 (1977)

27. Waltman, P., Hubbell, S. P., Hsu, S. B.: Theoretical and experimental investigations of microbial competition in continuous culture. In: Burton, $T$. (ed) Modeling and differential equations in biology. New York: Marcel Dekker 1980

Received July 20/Revised November 30, 1982 